\title{
Resection Urethroplasty for Urethral Stricture: Preliminary Findings from a Tertiary Care Hospital of Central Nepal
}

\author{
Sudeep Raj KC, ${ }^{1}$ Bhusan Raj Timilsina, ${ }^{1}$ Gaurav Devkota, ${ }^{2}$ Sulav Pradhan, ${ }^{1}$ Sabita Lamsal, ${ }^{3}$ Nirmal \\ Lamichhane $^{4}$ \\ ${ }^{1}$ Department of Urology, College of Medical sciences, Bharatpur, Nepal, ${ }^{2}$ Patan Hopsital, Kathmandu, Nepal, ${ }^{3}$ Chitwan \\ Medical College, Bharatpur, Nepal, ${ }^{4}$ B.P. Koirala Memorial Cancer Hospital, Bharatpur, Nepal.
}

\begin{abstract}
Background: Urethral stricture is relatively common problem that we encounter in urological practice. As urethral stricture causes progressive narrowing of the urethral lumen, signs and symptoms of urinary obstruction arise. These patients experience obstructive symptoms like poor stream, straining to urinate, incomplete voiding, end dribbling, urinary retention and recurrent urinary tract infections. Methods: A prospective cohort study was carried out in College of Medical Sciences, Chitwan, Nepal. All the patients who were operated with end to end anastomosis for urethral stricture with stricture length not more than $2.5 \mathrm{~cm}$ from January 2015 to June 2016 was included in this study. These patients were followed up for two year period for recurrence of the stricture and complications. Results: Mean age of patient in this study was 50.55 years, average length of stricture was $1.3 \mathrm{~cm}$. 66.67\% (12 patients) had complete success with no recurrence of the stricture. $33.33 \%$ (6 patients) had recurrence of stricture. Out of those 6 patients, 3 had undergone DVIU with no further recurrence of the stricture until the follow up period, one patient was treated with dilatation, two had complete failure and needed re-procedure. Conclusions: End to end anastomosis for short segment stricture had a fairly satisfactory result.
\end{abstract}

Keywords: DVIU; end to end urethroplasty; urethral stricture.

\section{INTRODUCTION}

Urethral stricture is a relatively common problem in men, which could result in a debilitating condition. As urethral stricture causes progressive narrowing of the urethral lumen, signs and symptoms of urinary obstruction arises. These patients experience obstructive symptoms like poor stream, straining to urinate, incomplete voiding, end dribbling, urinary retention and recurrent urinary tract infections. In general, the incidence of infection related to urethral stricture has decreased, especially in the developed world. ${ }^{1}$

One of the significant etiological factors for urethral stricture disease is trauma. Different types of trauma leading to urethral stricture are straddle injury, pelvic fracture - related urethral injury (PFUI) and iatrogenic. Straddle injury is the most common and typically occurs due to physical activities. ${ }^{2}$ From the studies, the average length of stricture of urethral stricture is $4.1 \mathrm{~cm}$ with most common site being the bulbar urethral $(52 \%)$, Lichen Sclerosis was reported as inflammatory strictures and the third leading cause of stricture disease $(26.6 \%)$, following idiopathic $(31.9 \%)$ and iatrogenic $(31.9 \%){ }^{3}$ In Brazil and Nigeria infection was responsible for urethral stricture in $15.2 \%$ and $66.5 \%$ of the cases respectively and $85 \%$ of times these were multi-location strictures. ${ }^{4,5}$ In urethral stricture, there is spongiofibrosis and with repeated urethral manipulations like urethral dilatation and DVIU, the length and density of spongiofibrosis increases. ${ }^{6}$ In the United States, direct-vision internal urethrotomy (DVIU) is the most commonly performed procedure for strictures less than $2 \mathrm{~cm}$. Nevertheless, the recurrence rate is much higher with this procedure. Resection urethroplasty has good outcome and is also cost effective compared to DVIU. ${ }^{7-9}$

From the management point of view, we take into consideration the length of the stricture, site and etiology of urethral stricture. For strictures of short segment, $<2 \mathrm{~cm}$ excision and an end to end anastomosis (resection urethroplasty) has been the ideal procedure which provides a good long-term results. ${ }^{10,11}$ The aim of this study is to evaluate the operative outcome of end-to-end urethroplasty in short segment stricture.

Correspondence: Dr. Sudeep Raj KC, Department of Urology, College of Medical Sciences, Bharatpur, Nepal. Email: sudeeprkc786@gmail.com. Phone: +977-9851030974. Orcid ID: http://orcid.org/0000-0002-6584-3768. DOI: $10.3126 / j \mathrm{cmsn} . v 15 i 1.22351$. Article Received:2018-07-17. Article accepted: 2019-02-18. 


\section{METHODS}

A prospective cohort study was carried out in College of Medical Sciences, Chitwan, Nepal from January 2015 to June 2016. A total of 60 patients who were treated for urethral stricture in 18 months. After selecting the patient which fulfilled the requirement of our inclusion and exclusion criteria, we had a total of 18 patients who under resection urethroplasty. Patients were evaluated with detail history, physical examination, urine analysis with urine culture. The retrograde urethrogram and micturating cystourethrogram were performed in every patients for diagnosis and treatment. Resection urethroplasty was performed under regional block (spinal and epidural anaesthesia). Inclusion criteria for end-to-end anastomosis were patients with urethral stricture, strictures less than $2.5 \mathrm{~cm}$, cases operated by single surgeon. Patients with long segment strictures of more than $2.5 \mathrm{~cm}$ and those who have undergone combined procedures like augmented anastomotic urethroplasty and re-do surgeries were not included in this study.

Resection urethroplasty was performed following the standard surgical protocols. Patients were placed in lithotomy position, complete mobilization of bulbar urethra and complete excision of strictured (fibrosed) segment was carried out. Then healthy urethral segments were speculated after which endto-end anastomosis was performed. Anastomosis was done in single layer using 5-0 polyglactin sutures in interrupted fashion. Following completion of dorsal suturing 14-Fr silicon catheter was placed and then ventral part was sutured. Peri neoscrotal area was sutured in layers with 3-0 polyglactin. Patients were hospitalized for about 3-5 days. Intravenous antibiotics (Ceftriaxone and Metronidazole) and analgesics were given. Wound dressing was done on 3rd post-operative day. After 5th POD, patients were discharged with catheter which was later removed in 14th post-operative day. In follow-ups, all the patient were evaluated with Uroflowmetry performed on 3rd, 6th and 12th month then annually thereafter. Retrograde urethrography and cystourethroscopy was done if patients developed voiding symptoms. Successful treatment was defined as no recurrence of stricture and good flow of urine in uroflowmetry. Treatment failure was defined as the need for any postoperative intervention including urethral dilation or DVIU. Fisher exact test was used to assess the significance of categorical risk factors for surgical failure and Student t-test was used to assess significance in continuous factors, e.g., age or operation time. Statistical significance was considered at $\mathrm{p}<0.05$. Data were entered in MSExcel and were imported to EZR software Version 3.4.1 for analysis

\section{RESULTS}

A total of 18 patients who fulfilled the inclusion criteria underwent resection urethroplasty. Out of which 11 patients( $61.11 \%$ ) had no recurrence of the stricture and had good surgical outcome. In six patients recurrence of the stricture was found, among which two patients presented with retention of urine (one in six months and other in 12 months). Foley's catheter could not be negotiated per urethra so retention was relieved with suprapubic catheterization. In the remaining four patients, diagnostic cystogram and cystoscopic findings were suggestive of short segment stricture which was less than $0.5 \mathrm{~cm}$. These patients were managed with DVIU (direct visual internal urethrotomy) among which three were managed successfully, remaining 1 patient did not improve with DVIU. The patient has been managed with monthly dilation and is having satisfactory flow rate and volume. In the total follow up period of 24 months one patient was lost in the follow up period after 12 months. There was no evidence of recurrence of the stricture therefore the patient is categorized as a success.

Mean operative time was $131.66 \pm 21.69$ mins ranging from 90 to 180 minutes. Mean excision length was $1.38 \pm 0.39$ ranging from 0.8 to $2.2 \mathrm{~cm}$. Length of stricture $1 \mathrm{~cm}$ and below was found in 5 patients, 1.1 to $1.5 \mathrm{~cm}$ in 9 patients and greater than $1.5 \mathrm{~cm}$ in 4 patients. Potential risk factor for the development of recurrence of the stricture was analyzed as shown in Table 1. There was no such risk factor identified in this study. $\mathrm{p}$ value was calculated using student t-test between success and failure group comparing the age and operative

\begin{tabular}{|c|c|c|c|}
\hline Factors & $\begin{array}{l}\text { Success } \\
(n=12)\end{array}$ & $\begin{array}{l}\text { Failure } \\
(n=6)\end{array}$ & $\begin{array}{l}\text { p- } \\
\text { value }\end{array}$ \\
\hline Age (years) & $\begin{array}{l}48.75000 \pm \\
20.83758\end{array}$ & $\begin{array}{l}54.16667 \pm \\
19.57975\end{array}$ & 0.604 \\
\hline Operative time (min) & $\begin{array}{l}129.1667 \pm \\
25.834555\end{array}$ & $\begin{array}{l}136.6667 \pm \\
9.309493\end{array}$ & 0.506 \\
\hline $\begin{array}{l}\text { Etiology } \\
\text { Traumatic } \\
\text { Non traumatic }\end{array}$ & $\begin{array}{l}6 \\
6\end{array}$ & $\begin{array}{l}2 \\
4\end{array}$ & 0.638 \\
\hline $\begin{array}{l}\text { Operative findings } \\
\text { Anterior urethral stric- } \\
\text { ture }\end{array}$ & 1 & 0 & \\
\hline $\begin{array}{l}\text { Bulbar urethral stric- } \\
\text { ture } \\
\text { Bulbomembranous } \\
\text { urethral stricture }\end{array}$ & 9 & 5 & 1 \\
\hline
\end{tabular}


KC et al. Resection Urethroplasty for Urethral Stricture: Preliminary Findings from a..

\begin{tabular}{|lllll|}
\hline \hline Table 2. Comparison of q max in follow up period. \\
\hline Uroflow & After 3 & After 6 & After 12 & After 24 \\
Q max & months & months & months & months \\
Good & 17 & 15 & 11 & 11 \\
& $(94.44 \%)$ & $(83.33 \%)$ & $(61.11 \%)$ & $(61.11 \%)$ \\
Bad & $1(5.56 \%)$ & 3 & $6(33.33 \%)$ & 6 \\
& & $(16.67 \%)$ & & $(33.33 \%)$ \\
Lost to & - & - & $1(5.56 \%)$ & $1(5.56 \%)$ \\
follow up & & & & \\
\hline
\end{tabular}

time which showed no statistical significance which was 0.604 and 0.506 respectively. Fisher exact test was used to compare etiology and site of stricture which also showed no statistical significance .

In early post-operative period three patients, experienced minor complication such as catheter, induced urinary tract infection and was managed with antibiotics. One patient had scrotal

\begin{tabular}{|lllll|}
\hline \multicolumn{3}{|l|}{ Table 3. Causes of urethral strictures in patients } \\
who underwent end-to-end anastomosis. \\
\hline \begin{tabular}{lllll} 
Etiology & Number & Percenage & & \\
Trauma & 8 & $(44.44 \%)$ & & \\
Iatrogenic & 6 & $(33.33 \%)$ & $\begin{array}{l}\text { Catheteri- } \\
\text { zation }=4\end{array}$ & TURP \\
& & & & \\
Idiopathic & 3 & $(16.67 \%)$ & & \\
Infection & 1 & $(5.56 \%)$ & & \\
\hline
\end{tabular}
\end{tabular}

hematoma which was managed conservatively. A total of six patients complained of pain in the scrotal area which was managed with analgesics. Two patients complained of decrease in the force of ejaculation and volume. One patient had erectile dysfunction and was managed with sildenafil citrate. No patient complained of penile shortening or curvature.

\begin{tabular}{|lll|}
\hline \multicolumn{3}{|l|}{ Table 4. Different site stricture. } \\
\hline Operative Findings & Number & Percentage \\
Anterior urethral stricture & 1 & $5.56 \%$ \\
Bulbar urethral stricture & 14 & $77.78 \%$ \\
$\begin{array}{l}\text { Bulbomembranous urethral } \\
\text { stricture }\end{array}$ & 3 & $16.67 \%$ \\
\hline
\end{tabular}

\section{DISCUSSION}

Surgical outcome can be influenced by many variables like length, severity and location of stricture. The surgical technique should be selected mainly according to stricture length, but we must also consider different factors like etiology and density of the spongiofibrosis. ${ }^{12}$ DVIU or end-toend urethroplasty is commonly accepted procedure for the treatment of short segment bulbar urethral stricture $(<2 \mathrm{~cm}) .9$ DVIU is recommended as the first choice of treatment when stricture is limited in a focal area. Stricture more than $1 \mathrm{~cm}$ in length, single setting of DVIU followed by end-to-end urethroplasty is commonly performed procedure. ${ }^{6,8,13}$ For a longer stricture $(>2 \mathrm{~cm})$,

ventral or dorsal onlay urethroplasty using a buccal mucosa graft is currently recommended, where the urethral lumen is relatively well preserved and the spongiofibrosis around the lumen is limited to 1 mm. ${ }^{14,15}$ Augmented anastomotic urethroplasty, with complete excision of the worst stricture segment, is currently recommended for strictures that cover a particularly dense and narrow area of 1 to $2 \mathrm{~cm}$ in length. Both ventral and dorsal onlay free grafts survive well with equal success rates. ${ }^{16,17}$

Short bulbar strictures are generally amenable to complete excision with primary anastomosis via a perineal incision, affording a high success rate of $95 \%$, as reported by Santucci et al. ${ }^{10}$ Eltahawy et al. ${ }^{18}$ published their series of 260 patients with bulbar stricture who underwent end-to-end anastomosis with a mean follow-up of 50.2 months. The stricture length ranged from 0.5 to $4.5 \mathrm{~cm}$ (mean $1.9 \mathrm{~cm}$ ) and the authors reported a success rate of $98.8 \%$. Recently, Barbagli et al. described a success rate of $90.8 \%$ in 153 patients who underwent Bulbar end-to-end anastomosis with a mean follow-up of 68 months. ${ }^{11}$ Jezior and Schlossberg summarized the surgical outcomes of excision and primary anastomosis for bulbar stricture on the basis of major series reported in the literature. These series showed a success rate of $93 \%$ in 443 patients with a range of $65 \%$ to $100 \%$ between series. ${ }^{19}$ Jun-Gyo Suh in 2013 studied 33 patients with bulbar stricture, end-to-end anastomosis had a success rate of $87.9 \%$ with a mean follow-up of 42.6 months. ${ }^{20}$

In this study, we studied a total of 18 patients with success rate $(66.67 \%)$ which is lower compared to other studies, probably because of early experience of the surgeon in the field of urethroplasty. Those patient who were categorized as a failure group did improve after a single setting of DVIU and dilatation. Two patients had complete failure as these patients presented with retention of urine and needed re-procedure.

Limitation of this study is small number of sample size, single surgeon and institutional study.

\section{CONCLUSIONS}

End-to-end anastomosis for short segment stricture is the procedure of choice and has better outcome.

\section{ACKNOWLEDGEMENTS}

The author would like to thank all the patients for giving consent to participate in the study.

Conflict of Interest: None 
KC et al. Resection Urethroplasty for Urethral Stricture: Preliminary Findings from a..

\section{REFERENCES}

1. Alwaal A, Blaschko S, McAninch J, B B. Epidemiology of urethral strictures. Transl Androl Urol. 2014;3(2):209-13.

2. Palminteri E, Berdondini E, Verze P, De Nunzio C, Vitarelli A, L C. Contemporary urethral stricture characteristics in the developed world. Urology. 2013;81:191-7.

3. Fenton AS, Morey AF, Aviles R, CR G. Anterior urethral strictures: etiology and characteristics. Urology. 2005;65(6):1055-8.

4. Mathur R, Aggarwal G, Satsangi B, Khan F, S $\mathrm{O}$. Comprehensive analysis of etiology on the prognosis of urethral strictures. International braz j urol. 2011 Jun;37(3):362-70. International braz j urol. 2011;37(3):362-70.

5. Ahmed A, GD K. Urethral stricture at Ahmadu Bello University Teaching Hospital, Zaria. East African medical journal. 1998;75(10):582-5.

6. Rourke KF, GH J. Primary urethral reconstruction: the cost minimized approach to the bulbous urethral stricture. The Journal of urology. 2005;173(4):1206-10.

7. Bullock TL, Brandes SB, Bullock TL, SB B. Adult anterior urethral strictures: a national practice patterns survey of board certified urologists in the United States. The Journal of urology. 2007;177(2):685-90.

8. Wright JL, Wessells $\mathrm{H}$, Nathens $\mathrm{AB}, \mathrm{W} \mathrm{H}$. What is the most cost-effective treatment for 1 to 2-cm bulbar urethral strictures: societal approach using decision analysis. Urology. 2006;67(5):889-93.

9. Barbagli G, Palminteri E, Lazzeri M, Guazzoni G, D T. Long-term outcome of urethroplasty after failed urethrotomy versus primary repair. The Journal of urology. 2001;165(6):1918-9.

10. Santucci RA, Mario LA, JW cA. Anastomotic urethroplasty for bulbar urethral stricture: analysis of 168 patients. The Journal of urology. 2002;167(4):1715-9.

11. Barbagli G, De Angelis M, Romano G, M L.
Long-term followup of bulbar end-to-end anastomosis: a retrospective analysis of 153 patients in a single center experience. The Journal of urology. 2007;178(6):2470-3.

12. Barbagli G, Guazzoni G, M L. One-stage bulbar urethroplasty: retrospective analysis of the results in 375 patients. european urology. 53(4). 2008:828-33.

13. Mangera A, C C. Management of anterior urethral stricture: an evidence-based approach. Current opinion in urology. 2010;20(6):453-8.

14. Barbagli G, Palminteri E, Guazzoni G, Montorsi F, Turini D, M L. Bulbar urethroplasty using buccal mucosa grafts placed on the ventral, dorsal or lateral surface of the urethra: are results affected by the surgical technique? The Journal of urology. 2005;174(3):995-8.

15. Patterson JM, CR C. Surgical techniques in substitution urethroplasty using buccal mucosa for the treatment of anterior urethral strictures. European urology. 2008;53(6):1162-71.

16. Guralnick ML WG. The augmented anastomotic urethroplasty: indications and outcome in 29 patients. The Journal of urology. 2001;165(5):1496-501.

17. Abouassaly R, KW A. Augmented anastomotic urethroplasty. The Journal of urology. 2007;177 (6):2211-6.

18. Eltahawy EA, Virasoro R, Schlossberg SM, McCammon KA, GH J. Long-term followup for excision and primary anastomosis for anterior urethral strictures. The Journal of urology. 2007; 177(5):1803-6.

19. Jezior JR, SM S. Excision and primary anastomosis for anterior urethral stricture. Urol Clin North Am. 2002;29:373-80.

20. Suh JG, Choi WS, Paick JS, SW K. Surgical outcome of excision and end-to-end anastomosis for bulbar urethral stricture. Korean journal of urology. 2013;54(7):442-7.

Citation: KC SR, Timilsina BR, Devkota G, Pradhan S, Lamsal S, Lamichanne N. Resection Urethroplasty for Urethral Stricture: Preliminary Findings from a Tertiary Care Hospital of Central Nepal. JCMS Nepal. 2019;15(1):1-4 